

\title{
In Situ Time-Resolved SAXS Study of the Formation of Mesostructured Organically Modified Silica through Modeling of Micelles Evolution during Surfactant-Templated Self-Assembly
}

Florentin Michaux, Niki Baccile, Marianne Impéror-Clerc, Luca Malfatti, Nicolas Folliet, Christel Gervais, Sabine Manet, Florian Meneau, Jan Skov Pedersen, Florence Babonneau

\section{- To cite this version:}

Florentin Michaux, Niki Baccile, Marianne Impéror-Clerc, Luca Malfatti, Nicolas Folliet, et al.. In Situ Time-Resolved SAXS Study of the Formation of Mesostructured Organically Modified Silica through Modeling of Micelles Evolution during Surfactant-Templated Self-Assembly. Langmuir, 2012, 28 (50), pp.17477-17493. 10.1021/la3038318 . hal-01457176

\section{HAL Id: hal-01457176 https://hal.sorbonne-universite.fr/hal-01457176}

Submitted on 6 Feb 2017

HAL is a multi-disciplinary open access archive for the deposit and dissemination of scientific research documents, whether they are published or not. The documents may come from teaching and research institutions in France or abroad, or from public or private research centers.
L'archive ouverte pluridisciplinaire HAL, est destinée au dépôt et à la diffusion de documents scientifiques de niveau recherche, publiés ou non, émanant des établissements d'enseignement et de recherche français ou étrangers, des laboratoires publics ou privés. 
IMPORTANT NOTE : Please be aware that slight modifications occurring after Proof correction may occur between this version of the manuscript and the version on the Publisher's website

In situ time-resolved SAXS study of the formation of mesostructured organically-modified silicas through modeling of micelles evolution during surfactant-templated self-assembly

Florentin Michaux, ${ }^{(1)}$ Niki Baccile, ${ }^{*(1)}$ Marianne Impéror-Clerc, ${ }^{(2)}$ Luca Malfatti, ${ }^{(1)}$ Nicolas Folliet, ${ }^{(1)}$ Christel Gervais, ${ }^{(1)}$ Sabine Manet, ${ }^{(2)}$ Florian Meneau, ${ }^{(3)}$ Jan Skov Pedersen, ${ }^{(4)}$ Florence Babonneau ${ }^{(1)}$

${ }^{1}$ UPMC Univ Paris 06, UMR 7574, Chimie de la Matière Condensée de Paris, F-75005, Paris, France

CNRS, UMR 7574, Chimie de la Matière Condensée de Paris, F-75005, Paris, France

Collège de France, UMR 7574, Chimie de la Matière Condensée de Paris, F-75005, Paris,

France

${ }^{2}$ Laboratoire de Physique de Solides, UMR 8502, Bât. 510, Université Paris-Sud, F-91405 Orsay, France

${ }^{3}$ SWING, Synchrotron Soleil, BP 48, F-91192 Gif-sur-Yvette, France

${ }^{4}$ Department of Chemistry and iNANO Interdisciplinary Nanoscience Center, Aarhus University, DK-8000 Aarhus, Denmark

\section{Abstract}


The mechanisms of formation of organically-modified (phenyl, vinyl, methyl) silica materials with cubic $\mathrm{Pm}^{\mathbf{3}} n$ and hexagonal $p 6 m$ periodic mesostructures obtained in one-step in the presence of the cetyltrimethylammonium bromide $\left(\mathrm{CTA}^{+} \mathrm{B}\right)$ surfactant is reported in this study. Understanding the way these complex materials form is difficult but undoubtedly necessary to control the material structure and its properties due to the combined presence of surface organic groups and large surface areas. Here, the mechanism of formation is clarified based on the modeling of time resolved in situ Small Angle X-ray Scattering (SAXS) experiments, with a specific focus on the micelles evolution during the material formation. Their fast self-assembly is followed for the first time with a quick temporal resolution of a few seconds using a third generation synchrotron radiation source. To better understand the behaviour of the complex organically-containing mesostructure, we perform a comparative study with the corresponding, organo-free, isostructural materials obtained from three different surfactants $\left(\mathrm{CTA}^{+}, \mathrm{CTEA}^{+}\right.$and $\left.\mathrm{CTPA}^{+}\right)$having constant chain length $\left(\mathrm{C}_{16}\right)$ and increasing polar head volume (Met-, Et-, Prop-). Numerical modeling of SAXS data was crucial to highlight a systematic sphere-to-rod micellar transition, otherwise undetected, before the formation of the 2D-hexagonal phase in both organo-free and organo-containing systems. Then, two different pathways are found in the formation of the cubic $\mathrm{Pm}^{\overline{\mathbf{3}}} n$ mesostructure: either an ordering transition from concentrated flocs of spherical micelles

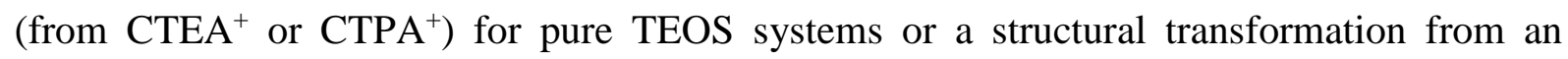
intermediate 2D-hexagonal mesophase in organosilane systems (from $\mathrm{CTA}^{+}$). Combining the comparison between organo-free and organo-containing systems with numerical modeling, we find that the hexagonal-to-cubic phase transition in the organically-modified materials seems to be strongly influenced not only by the obvious presence of the organic group but also by a quicker and more massive condensation kinetics of silicate oligomers on the $\mathrm{CTA}^{+}$ 
micellar surface. Finally, quite unexpectedly, we find a wormlike-to-sphere, micellar transition in the $\mathrm{CTPA}^{+}$system.

\section{Introduction}

Hybrid organic-inorganic silica-based materials obtained through solution precipitation using sol-gel chemistry have been more and more studied in the past 25 years because of their number of applications (e.g., tuning of the hydrophilic/hydrophobic nature of the surface, incorporation of luminescent hosts, metal complexing, mechanical properties...), as recently discussed. ${ }^{1-3}$ For instance, phenyl groups are used to enhance silica hydrophobic character while vinyl ones can be introduced as specific sites for further chemical grafting ${ }^{4-6}$ thus enhancing, among others, catalytic, sensing, molecular recognition, luminescence properties. The distribution and accessibility of the organic functions can be tuned by adding and controlling mesoscale porosity in the material's bulk. ${ }^{7-9}$ Two approaches exist to achieve this goal, either using the post-grafting route on an existing porous network or the cocondensation method, in which the organically-modified precursor reacts in the presence of the porogen. ${ }^{1,3,10-13}$ Here, we investigate the co-condensation method.

From the point of view of pore organization, 2D and 3D networks are classically obtained. Interestingly, in organo-free systems, mesostructures are generally controlled through the use of a surfactant with a given molecular geometry. This is not the case when an organic group is employed. For instance, SBA-3 and SBA-1 refer to well-known materials with 2D-hexagonal p6m and cubic mesostructure, respectively. The former is obtained using cetyltrimethylammonium bromide (CTAB) as porogen while the latter can be made from cetyltriethylammonium bromide (CTEAB). On the contrary, the simple presence of a phenyl 
group in a CTAB-based system is enough to provide a cubic $P m^{\mathbf{3}} n$ mesostructures, ${ }^{14,15}$ which is not obtained with CTAB alone using the precipitation method. The change in mesostructure has been explained using arguments related to the "packing parameter" $g$, first introduced by Israelachvili et al. ${ }^{16}$ to correlate the molecular geometry of the surfactant in water with the corresponding micellar curvature. The parameter $g=V /\left(A_{0 .} h\right)$ is related to the total volume, $V$, of the surfactant hydrophobic part, the surface per polar head, $A_{0}$, and the length of the surfactant tail, $h$. This parameter has also been proposed to explain at the molecular level the different liquid crystal structures (lamellar, hexagonal or cubic). For example, the increase of the polar head size from CTAB to CTEAB leads to the decrease of $g$ and thus to the formation of more stable spherical micelles which promote a micellar cubic phase instead of a 2Dhexagonal one. ${ }^{8}$ Nevertheless, coming back to the formation of templated materials, despite some evident correlation between the $g$ value and the type of mesophase, the final structure also strongly depends on other parameters like the type of counter-ions in presence or the synthesis temperature. For sake of clarity, the molecular structure and the corresponding gparameter are shown in Figure 1.

In this sense, the use of an organically-modified silica precursor also showed to have a very strong influence on the structure of the final mesophase, ${ }^{4-6,17-19}$ thus making practically impossible to predict in any way whatsoever the type of pore organization when the chemical complexity of the system increases. This is clear when phenyl-modified alkoxysilanes are employed using CTAB as porogen. In this case, a phase transition between a hexagonal p6m phase and a micellar cubic $\mathrm{Pm}^{\overline{\mathbf{3}}} n$ mesostructure was reported ${ }^{14}$, 15, suggesting specific interactions between the phenyl groups and the polar head of the surfactant. Similar behaviors were found for mixed CTAB/CTEAB systems in absence of organo-modified alkoxysilanes. In this context, the first step is to investigate the behavior of organosilane-free systems, where the nature of the surfactant alone (CTAB, CTEAB or CTPAB) induces the change in 
mesophase. Then, the more complex organosilane-containing systems can be understood as the organic group of the alkoxysilane strongly influences the micellar curvature.

The relationship between the resulting mesophase and the surfactant used and/or the synthesis conditions (counter-ions, temperature) starts to be understood for some specific systems like MCM-41 and SBA-15. For these materials, the steps involved during the structural transition from micelles in solution to the final mesophase have been largely explored. The formation mechanism of the mesostructure has been studied using spectroscopic techniques, ${ }^{20-25}$ transmission electron microscopy ${ }^{26,27}$ and small angle scattering measurements. ${ }^{26,28-37}$ The suitability of these experimental methods to understand the mesophase formation have been discussed in a previous paper. ${ }^{38}$ Most of these studies were mainly done ex-situ, which may induce modifications in such quickly evolving systems. For this reason, the most appropriate methods are based on in situ small angle scattering techniques coupled with a high-power source. In this sense, small angle X-ray scattering using synchrotron radiation allows very short accumulation time (as low as 0.5 seconds), which is essential for a good description of fast reactions kinetics.

Thus far, most in situ SAXS experiments on the formation of mesostructured materials mainly focused on the study of the Bragg reflections intensities and positions to better follow, and consequently understand, the evolution of the mesophase as a function of the reaction time. Unfortunately, this approach is not enough to precisely describe the evolution of micellar shape with time and, in this sense, is exploited far below its true capabilities. To go beyond, and appreciate at best this powerful technique, several conditions should be fulfilled, like long reaction times ( hours) that help to better visualize the micellar signal, for which the electron density contrast with water is lower using X-rays with respect to neutrons. In addition, efficient and well-adapted modeling of the SAXS intensity taking into account as many 
components as possible (e.g., shape factors of micelles, structure factor for interacting micelles, smearing of the micellar palisade, background...) is required.

This approach was successfully used to solve a long-dated debate in literature concerning the mechanism of formation of the well-known Pluronic P123-templated SBA-15 materials, for which a sphere-to-rod transition mechanism due to the interaction between silica oligomers and micelles in water prior to the formation of the final 2D-hexagonal $p 6 m$ mesophase is now established. $^{30,31,33,36,39}$

In the present work, we apply the above-described approach which employs timeresolved in situ Small Angle X-ray Scattering (SAXS) experiments using Synchrotron radiation to study the mechanism of formation of organically-modified silica powders obtained by precipitation with three different organosilanes (methyltriethoxysilane-MTES, vinyltriethoxysilane-VTES and phenyltriethoxysilane-PTES) using CTAB as surfactant (Figure 1). Compared with previous in situ SAXS studies, this work goes several steps further both in terms of the complexity of the chemical environment and in terms of the technique itself. First of all, we include chemical heterogeneity, as organosilanes display very different reactivity than classical TEOS and organic groups can dynamically interfere with the local micellar curvature; this point causes time-dependent transitions between mesophases, such as from the 2D-hexagonal to cubic $\mathrm{Pm}^{\overline{\mathbf{3}}} n$ for phenyl-modified organosilanes. ${ }^{14,}{ }^{15}$ Finally, the experimental conditions impose very short reaction times ( minutes), which make the acquisition of good quality signal very hard, requiring the use of a synchrotron source.

To approach this study, we initially bring more insight on a model system composed of micellar solutions of three closely related cationic surfactants with the same alkyl chain $\left(\mathrm{C}_{16}\right)$ and different polar heads of increasing sizes (Met-, Et-, Prop-), CTAB, CTEAB and CTPAB, and using TEOS alone as silica source (Figure 1). Under these conditions, materials with 2Dhexagonal (CTAB) and cubic $\mathrm{Pm}^{\overline{\mathbf{3}}} n$ (CTEAB, CTPAB) mesostructures are obtained and at 
the same time we fill the gap existing on their mechanism of formation. In the end, we show how the strength of combining fast SAXS experiments obtained on a third generation synchrotron source to numerical modeling to study the micellar shape evolution observed on these "simpler" systems helps understanding the formation of more complex, isomorphic, hybrid materials obtained from a mixture of TEOS and an organically-modified silane.

\section{Experimental methods}

\section{Surfactants:}

Three cetyltrialkylammonium bromide surfactants have been used as template. They differ from the size of the alkyl chains linked to the ammonium headgroup, i.e. methyl for CTAB $\left(\mathrm{C}_{16} \mathrm{H}_{33} \mathrm{~N}^{+}\left(\mathrm{CH}_{3}\right)_{3} \mathrm{Br}^{-}\right)$, ethyl for CTEAB $\left(\mathrm{C}_{16} \mathrm{H}_{33} \mathrm{~N}^{+}\left(\mathrm{C}_{2} \mathrm{H}_{5}\right)_{3} \mathrm{Br}^{-}\right)$and propyl for CTPAB $\left(\mathrm{C}_{16} \mathrm{H}_{33} \mathrm{~N}^{+}\left(\mathrm{C}_{3} \mathrm{H}_{7}\right)_{3} \mathrm{Br}^{-}\right)$. CTAB was purchased from Aldrich and CTEAB and CTPAB were synthesized according to the method described by Menger et al. ${ }^{40}$

\section{Synthesis of silica-based materials:}

All samples were prepared in acidic aqueous solutions using tetraethoxysilane (TEOS, Aldrich) or mixture of TEOS and methyltriethoxysilane (MTES), vinyltriethoxysilane (VTES) or phenyltriethoxysilane (PTES). The molar ratio among all reactants (inorganic precursor/surfactant $/ \mathrm{HCl} / \mathrm{H}_{2} \mathrm{O}$ ) is adapted from the original procedure of Huo et al. ${ }^{8}$ and equals $1 / 0.12 / 9.2 / 200$. The materials synthesis procedure is detailed elsewhere. ${ }^{41}$ In the case of mixture of precursors, the molar ratio between TEOS and organosilane (MTES, VTES and PTES) is fixed to 4:1 and surfactant used is CTAB.

\section{Experimental setup:}


SAXS measurements were carried out at the SWING SOLEIL beamline (energy $12 \mathrm{keV}$ ). The sample-CCD camera distance was $1.50 \mathrm{~m}$, and the q range was $6.0 \cdot 10^{-3}-0.7 \AA^{-1}$. The q range calibration was made using a silver behenate standard sample $\left(\mathrm{d}_{\mathrm{ref}}=58.38 \AA\right)$.

For the absolute intensity calibration, scattering patterns of the empty capillary and the capillary filled with deionized water were always recorded first. The value of the constant intensity contribution of water is equal to $0.016 \mathrm{~cm}^{-1}$ on absolute scale. Then, the signal of the same capillary filled with the solvent solution was recorded for subtraction purposes before the introduction of the synthesis solution. During each kinetics experiment, the capillary was kept fixed inside the beam using a capillary holder, so that all patterns were recorded at the same position. The experimental setup is described more accurately in previous studies. ${ }^{29}, 30$, 36,39

For SAXS, the scattering length density is the product of the classical radius of the electron $r_{e}$ $=2.81794 .10^{-15} \mathrm{~m}$ and the electron density (number of electrons per unit volume). In the tables, we give the electron density values in e/nm ${ }^{3}$ rather than the scattering length density, but the coefficient $r_{e}$ is taken into account for the absolute scaling (in $\mathrm{cm}^{-1}$ ) in the intensity expression. The electron density of the solvent $\left(\mathrm{H}_{2} \mathrm{O}+\mathrm{HCl}\right)$ is equal to $350 \mathrm{e} / \mathrm{nm}^{3}$. For the three surfactants used, the estimated values of the electron densities are $280 \mathrm{e} / \mathrm{nm}^{3}$ for the hydrophobic moiety and respectively 466, 432 and $392 \mathrm{e} / \mathrm{nm}^{3}$ for $\left(\mathrm{CH}_{3}\right)_{3} \mathrm{~N}^{+} \mathrm{Br}^{-}$(CTAB), $\left(\mathrm{C}_{2} \mathrm{H}_{5}\right)_{3} \mathrm{~N}^{+} \mathrm{Br}^{-}(\mathrm{CTEAB})$ and $\left(\mathrm{C}_{3} \mathrm{H}_{7}\right)_{3} \mathrm{~N}^{+} \mathrm{Br}^{-}(\mathrm{CTPAB})$ hydrophilic polar heads. Nevertheless, due to the high concentrations of $\mathrm{HCl}$ in the solution for both systems, a counter-ion exchange may occur, and thus, the presence of $\mathrm{Cl}^{-}$instead $\mathrm{Br}^{-}$in the polar head must be taken into account. In this case, the electron densities of the hydrophilic parts become 380, 357 and 351 e/nm ${ }^{3}$ for respectively $\left(\mathrm{CH}_{3}\right)_{3} \mathrm{~N}^{+} \mathrm{Cl}^{-}$CTAC, $\left(\mathrm{C}_{2} \mathrm{H}_{5}\right)_{3} \mathrm{~N}^{+} \mathrm{Cl}^{-}$CTEAC and $\left(\mathrm{C}_{3} \mathrm{H}_{7}\right)_{3} \mathrm{~N}^{+} \mathrm{Cl}^{-}$ CTPAC. For sake of simplicity, the three surfactants are called $\mathrm{CTA}^{+}, \mathrm{CTEA}^{+}$and $\mathrm{CTPA}^{+}$in the following parts of the article. 


\section{Fitting models:}

The SAXS results have been interpreted using models previously reported in the literature ${ }^{33 \text {, }}$ 34, 36 developed in the case of the formation of SBA-15 materials. All data have been treated with a least-square fitting procedure. Notations for these models are summarized in this paragraph. The intensity is modeled by the sum of the following contributions:

$$
I_{\text {tot }}(q)=I_{\text {micelle }}(q)+I_{\text {cluster }}(q)+I_{\text {Bragg }}(q)+I_{\text {Porod }}(q)
$$

$I_{\text {micelle }}(q)=A P(q) S(q)$, where $P(q)$ is the form factor of the micelles that can be either spherical, ellipsoidal, cylindrical or wormlike, and which are all described using a core/shell model with a core radius $R_{c}$, a total radius $R_{\text {tot }}$, an ellipticity $\varepsilon$ (of, respectively, ellipsoid or cross section for cylindrical or wormlike micelles), smearings $\sigma_{\text {core }}$ and $\sigma_{\text {shell }}$ of the core and shell interfaces, a contrast term $\alpha=\left(\rho_{\text {shell }}-\rho_{0}\right) /\left(\rho_{\text {core }}-\rho_{0}\right)$, where $\rho_{0}, \rho_{\text {shell }}$ and $\rho_{\text {core }}$ stand for the electronic density of, respectively, the solvent, the hydrophilic shell and the hydrophobic core, and a polydispersity $\sigma$ of the radii. The resulting typical electron density profile for this eore/shell model with smearing of both interfaces is depicted in Figure 3. Other relevant parameters for the wormlike micelles model, $L_{\text {cont }}$ and $b_{K u h n},{ }^{42}$ (overall contour length and Kuhn length) are introduced directly in the text (in section 3.4.). $S(q)$ is the structure factor associated with the micelles in solution or within flocs. $I_{\text {Bragg }}(q)$ is the contribution of the Bragg peaks from the long-range ordered mesophase. For the 2D-hexagonal mesophase, $I_{\text {Bragg }}(q)=B P(q)\left[1-\beta(q) G(q)+\beta(q) G(q) Z_{0}(q)\right]{ }^{36}$ where $B$ is the scaling factor, $Z_{0}(q)$ the Bragg peaks intensity without disorder, and $\beta(q)$ and $G(q)$ are the two disorder terms. The fit parameters are the core/shell cylindrical form factor $P(q)$ of the micelles ordered within the mesophase, the lattice parameter $a$, the average length $L$ and diameter $D$ of the grains, the polydispersity $\sigma$ (term $\beta(q)$ ) and the Debye-Waller $\sigma_{D W}$ disorder parameter (term $G(q)$ ). 
This form factor $P(q)$ for the mesophase is the same as for the free cylindrical micelles (if any in coexistence with the mesophase).

The silica clusters contribution $I_{\text {cluster }}(q)=2 C \frac{\exp \left(-q^{2} R_{g}^{2}\right)-1+q^{2} R_{g}^{2}}{\left(q^{2} R_{g}^{2}\right)^{2}}$ is modeled using the Debye expression of a polymer chain, with a gyration radius $R_{g}$ (typically 2-5 $\AA$ ) and its overall intensity is given by the constant $C$. Lastly, the Porod contribution $I_{\text {Porod }}(q)=\frac{K}{q^{4}}$ takes into account the scattering by the interface of the grains of the material or a large-scale inhomogeneity, and contributes only in the low- $q$ region.

\section{Results and discussion}

Temporal evolutions of I(q) as a function of time for TEOS mixed with MTES, VTES and PTES using $\mathrm{CTA}^{+}$as surfactant in acidic water are presented on Figure 2. The use of a mixture of TEOS and VTES leads to a 2D-hexagonal mesophase with three resolved peaks after 150 seconds of reaction as seen on the corresponding 2D plot in Figure 2. Between 400 and 500 seconds after the addition of the silica source, the intensity of the (10) reflection decreases tightly, the (11) and (20) disappear and two other diffraction peaks which can be indexed as the (200) and (210) reflections of a cubic $\mathrm{Pm}^{\overline{\mathbf{3}}} n$ mesophase appear. In this case, the (10) reflection of the 2D-hexagonal phase becomes the (211) reflection of the cubic phase through an epitaxial transformation. Based on this relationship, Terasaki and coworkers have proposed a model to explain the phase transition: the hexagonal and cubic structures are related, respectively, by their [001] and [111] directions. The epitaxial relationship is obtained by a periodic modulation of the curvature along the cylinders longest axis. Finally, cylinders split into spherical micelles composing the cubic phase. ${ }^{43}$ This kind of transformation from a 
2D-hexagonal phase to a cubic $\mathrm{Pm}^{\overline{\mathbf{3}}} n$ one with an epitaxial relation between the (10) and (211) reflections have already been reported in other systems like for example a system composed of $\mathrm{CTEA}^{+}$with trimethylbenzene (TMB) in $\mathrm{HCl}$ water solution using TEOS as inorganic precursor. ${ }^{43,} 44$ The situation is almost similar in the case of a mixture of TEOS and PTES, even if there is no sharp decrease of the intensity of the (10) reflection at the moment when the Bragg peaks for the cubic phase appear. The same epitaxial relation between the (10) reflection of the hexagonal phase and the (211) one of the cubic is observed and the higher intensities of the diffraction peaks compared to the VTES/TEOS system allow seeing this correspondence also between the second order reflections (20) and (422). The relative intensities of the diffraction peaks reflect a coexistence of the 2D-hexagonal phase and the cubic one in the case of PTES/TEOS at the end of the kinetics, unlike for the VTES/TEOS system where a pure $\operatorname{Pm}^{\overline{\mathbf{3}}} n$ is obtained at the end. This kind of transformation from a mesostructured phase to another one without dissolution-reprecipitation process has already been reported, from 2D-hexagonal to lamellar as a function of the reaction time ${ }^{45,46}$ and from lamellar to 2D-hexagonal ${ }^{47}$ or $2 \mathrm{D}$-hexagonal to cubic $I a 3 d^{48}$ with an increase of the temperature. Nevertheless, an epitaxial correlation between the two ordered phases is rarely encountered. It seems that the driving force of these phase transitions is the polymerization of the silica species all along the synthesis. For the MTES system, the kinetics of cubic mesophase formation is slower and in the experiments presented here, only the peak of the hexagonal phase alone can be clearly identified.

Since the same hexagonal and cubic mesophases can be obtained on the corresponding organo-free mesostructured materials but employing $\mathrm{C}_{16}$ surfactants with increasing polar head volume, as shown on the corresponding XRD patterns in Figure S1 in the Supporting Information, it becomes crucial to compare data presented in Figure 2 with a detailed study performed on simpler systems with pure TEOS as silica source but different surfactants. 
Figure 1 shows the chemical structures of CTAB, CTEAB and CTPAB, for which, in terms of $g$-values, one can calculate 0.32 for CTAB, 0.26 for CTEAB, 0.21 for CTPAB (detail is given in the Supporting Information). Considering the evolution of the $g$-parameter, one could expect that the micellar curvature increases from CTAB to CTPAB. To a first sight, this can appear correct, however, this work shows that the behavior is in fact much more complicated and that simple assumptions based on the $g$-parameter alone appear to be oversimplified. For this reason, we approach the study of $\mathrm{CTA}^{+}$templated organo-silica materials in the following way: the formation of SBA-3 (CTA ${ }^{+}$with pure TEOS) is described in section 3.1., followed by SBA-1 (CTEA ${ }^{+}$with pure TEOS) in section 3.2. Section 3.3. is devoted to the study of the hybrid organically-modified silica materials $\left(\mathrm{CTA}^{+}\right.$with mixtures of TEOS and an organosilane (MTES, VTES and PTES) compared to the organosilane-free materials. Lastly, in section 3.4., the unexpected micellar behavior of CTPA ${ }^{+}$with the formation of wormlike micelles is specifically underlined.

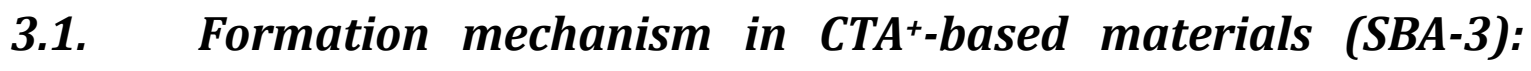 Cylindrical vs. spherical micelles}

The structure of the micelles formed by $\mathrm{CTA}^{+}$in acidic water has been investigated prior to the addition of the silica source. The corresponding scattering curve is presented in Figure 3 and has been fitted using an ellipsoidal core-shell model with smearing of both interfaces and depicted at the top of Figure 3 (fit parameters shown in Table S.1). Best fit corresponds to prolate ellipsoidal micelles with a weak ellipticity of 1.9 . The core radius $R_{C}$ equals $18 \AA$. The shell thickness equals $2 \AA$ and the smearings of the electronic density at the interfaces are the same $\left(\sigma_{\text {core }}=\sigma_{\text {shell }}=1 \AA\right)$. The contrast term $\alpha(-0.3)$ is negative and close to 0 , which means that the electronic density level of the hydrophilic shell is close to the solvent ones, suggesting the presence of $\mathrm{Cl}^{-}$anions within the shell instead of $\mathrm{Br}^{-}$ones (see the electron density profile 
for $\mathrm{CTA}^{+}$in Figure 3), as previously discussed. ${ }^{41}$ This micelles structure established from the fits is similar to those reported in the literature for $\mathrm{CTA}^{+}$systems determined by SAXS or SANS. ${ }^{49-57}$ It should be mentioned here that the fit is restricted to q values greater than $0.02 \AA^{-}$ ${ }^{1}$, to avoid the low-q region where an extra contribution to the intensity is measured, whose origin is not understood. Interestingly, no interaction peak is observed, as one would expect from this system at the concentration used here $\left(3.10^{-2} \mathrm{M}\right){ }^{52}$ This is easily explained by a screening effect of the electrostatic interactions from the large amount of ions present $\left(\mathrm{Cl}^{-}\right.$in solution, from $\mathrm{HCl}$, is $2.4 \mathrm{M}$ ), as reported for example by Aswal et al. ${ }^{56}$ Moreover, the total counter-ion exchange at the micellar surface from $\mathrm{Br}^{-}$to $\mathrm{Cl}^{-}$is also detected. Indeed, a broad peak related to the contribution of the outer shell of the micelles to the form factor is reported when $\mathrm{Br}^{-}$is present at the micelles surface due to its high electronic density. ${ }^{56}$ On the opposite, the contrast between the shell and the hydrophobic core is reduced if $\mathrm{Cl}^{-}$is present around the micelles because of its lower electronic density. Then, this leads to a plateau at low $\mathrm{q}$ instead of a broad peak, which is the case in our results.

Upon addition of the inorganic precursor, TEOS, a 2D-hexagonal mesophase is formed and the first diffraction peaks appear after $200 \mathrm{~s}$ (Figures 4, 5, 6 and S2) and up to five different peaks are recorded. The cell parameter of the 2D-hexagonal mesophase decreases as a function of the reaction time from 49.4 to $48.7 \AA$. This evolution is expected, and has been previously reported for MCM-41 and SBA-15 type materials, due to an increase of the silica condensation which leads to a shrinkage of the hexagonal structure. One should notice that the intensity of the (11) reflection is very weak until $400 \mathrm{~s}$ (Figures 4 and 5) and is still lower than the (20) one all along the kinetic measurement. This observation has been already made for MCM-41 materials ${ }^{45}$ and is directly related to the form factor of the surfactant cylinders which presents a minimum in the same q range as the $(11)$ position $(P(q)$ in Figure 6). 
The analysis of the micelles contribution before the appearance of the Bragg peaks (until 200 s) happens to be more difficult and we try to approach it hereafter using a best fit procedure. It is highly expected that a sphere-to-rod transition takes place simply because the resulting final mesophase is 2D-hexagonal. In the case of SBA-15 for instance ${ }^{31,36}$ the formation of cylindrical micelles is detected by a clear visual change in the form factor of the micelles upon time during the in situ SAXS study. The cylinder form factor is detected by the presence of a $\mathrm{q}^{-1}$ slope at low-q. In this case, though, the time-resolved in situ SAXS experiments (Figure 5) for $\mathrm{CTA}^{+}$do not show a $\mathrm{q}^{-1}$ slope upon time, hence it is then more difficult to choose between a spherical or a cylindrical model for the structure of the micelles, as detailed in the following.

The scattering curves are interpreted in terms of four different contributions, according to a previous model which has already been introduced in SBA-15 in situ SAXS studies ${ }^{36}$ and presented before: $I_{\text {tot }}(q)=I_{\text {micelle }}(q)+I_{\text {cluster }}(q)+I_{\text {Bragg }}(q)+I_{\text {Porod }}(q)$. The first contribution $I_{\text {cluster }}(q)$ is attributed to the formation upon time of silica clusters by the condensation reaction and its intensity is then proportional to the condensation rate. This contribution to the scattered intensity provides a constant value at low-q and exhibits a I(q) $\mathrm{q}^{-2}$ (log-log scale) behavior for $\mathrm{q}>0.2 \AA^{-1}$. $I_{\text {cluster }}(q)$ (green line in Figure 6 and S2) becomes quite large at the end of the kinetics. The radius of gyration $R_{g}$ of these silica clusters is about 2-5 Ångströms and is increasing upon time, in agreement with the ongoing silica condensation. The second contribution $I_{\text {Porod }}(q)$ gives an increase of the intensity only at low q values $\left(\mathrm{q}<0.016 \AA^{-1}\right)$, that can be modeled by a $1 / \mathrm{q}^{4}$ Porod slope. It corresponds to the scattering by the surface of large scale grains of inhomogeneous size within the synthesis batch solution, like non hydrolyzed TEOS droplets at the beginning of the reaction and/or the surface of the silica grains after precipitation of the mesophase. The intensity of this contribution exhibits 
sometimes large fluctuations upon time, because during one individual exposure time of $0.5 \mathrm{~s}$, the composition of the synthesis solution exposed to the X-ray beam is itself varying a lot. Then, the major structural information can be deduced essentially from the two last contributions $I_{\text {micelle }}(q)$ and $I_{\text {Bragg }}(q)$, in the mid-q range between 0.016 and $0.35 \AA^{-1}$. A close analysis of the $\mathrm{CTA}^{+}$system shows that $I_{\text {micelle }}(q)$ is decreasing upon time, and which can be interpreted as a decrease of the micelles number in solution explained by their gradual consumption (or the consumption of surfactant) to form the 2D-hexagonal mesophase, that appears after 200 seconds. One should notice that after 500 seconds of reaction $I_{\text {micelle }}(q)$ equals zero and only $I_{\text {Bragg }}(q)$, the mesophase signal, is present, along with the two other contributions $\left(I_{\text {cluster }}(q)\right.$ and $I_{\text {Porod }}(q)$ ) (Figure S2). Between 150 and $500 \mathrm{~s}, I_{\text {micelle }}(q)$ is weak compared to the other signals, so it is difficult to detect unambiguously the modifications of the shape or size of the free micelles upon time, in particular if they are spherical or rod-like. A comparison with the formation of SBA-15 2D-hexagonal materials is necessary to better understand the unexpected behavior of SBA-3 reported here. SBA-15 is prepared from spherical micelles of P123 and the addition of the inorganic precursor leads to a change of the scattering signal from a plateau to a $\mathrm{I}(\mathrm{q}) \sim \mathrm{q}^{-1}$ slope, characteristic of a transition from spherical to isolated cylindrical micelles, ${ }^{31,36}$ indicating that the cylindrical micelles pack together to form the hexagonal mesophase. This modification of the scattering curves shape should also be detected in the equivalent q range for the $\mathrm{CTA}^{+}$system since the micelles structure before the addition of the silica source and the ending mesophase are the same as for SBA-15 and SBA-3. At a first sight, the behavior of the SBA-3 system in acidic water is different since the intensity is essentially constant in the q-range between 0.016 and $0.6 \AA^{-1}$ during the synthesis. It seems that no modification of the micelles shape occurs after the addition of the inorganic precursor and that no cylindrical micelles are formed prior to the 
formation of the mesophase. In fact, the entire set of data from the curve recorded before the addition of the silica source to the micelles signal present under the Bragg peaks during the reaction until 500 seconds can be fitted using a core-shell spherical/ellipsoidal micelles model (data not shown). The presence of spherical micelles all along the reaction without a transition towards cylinders is in good agreement with the so-called "reservoir" mechanism reported for MCM-41 synthesis by Patarin et al..$^{20,21}$ They describe the micelles as surfactant reservoirs and reported that the mesophase is formed from interactions between the surfactant monomers in solution and the silica species. Then, the number of free surfactant monomers in solution decreases and $\mathrm{CTA}^{+}$molecules leave the micelles to restore the micelles/monomers equilibrium defined by the critical micellar concentration. This proposed mechanism, determined from fluorescence probing technique, is based on the study of the ion exchange between the surfactant counter-ion $\left(\mathrm{Br}^{-}\right.$or $\left.\mathrm{Cl}^{-}\right)$and silicate ions around the micelles of surfactant. They showed that the silicate species do not accumulate around the micelles, thus not acting directly as template. In this mechanism, no change in the micelles structure occurs and their number decreases in parallel to the growth of the mesophase. This is in good agreement with our experimental observations even if the synthesis conditions are different (basic vs. acidic conditions here). Nevertheless, from the appearance of the first Bragg peak after 200 seconds of reaction, in the framework of the "reservoir" mechanism, the system would be composed of the coexistence of free globular (spherical or ellipsoidal) micelles and of surfactant cylinders packed in a hexagonal array. As already mentioned, it is rather expected that the shape of the micelles evolves upon time. Then we propose another mechanism, which accounts for a sphere-to-rod mechanism. To do so, a careful analysis of the form factor of the 2D-hexagonal mesophase is first necessary. Indeed, the form factor $P(q)$ of the surfactant cylinders can be deduced from the relative intensities of the (11), (20) and (21) reflections recorded at the end of the measurement (Figure 6). The lower intensity of the (11) 
reflection compared to the (20) comes from a minimum of the form factor $P(q)$ at the corresponding q value (blue line in Figure 6). This allows to determine the size of the cylinders cross section very precisely (hydrophobic radius and thickness of the hydrophilic shell) and also the value of the contrast term $\alpha$ (Table in Figure 6 and profiles in Figure S2), where $\alpha=\left(\rho_{\text {shell }}-\rho_{0}\right) /\left(\rho_{\text {core }}-\rho_{0}\right)$. The hydrophobic radius and the shell thickness are equal respectively to 16.9 and $6.1 \AA$, along with a large smearing of $5.75 \AA$ of the outer shell. The cell parameter determined from the position of the (10) reflection is equal to $48.8 \AA$. By comparison with the cylinders diameter, a $2.8 \AA$ thick wall separates two adjacent surfactant cylinders. The contrast term $\alpha$ is close to -1 , in agreement with the presence of silica species incorporated in-between the micelles within the material. This combination of parameters leads to a specific shape of the form factor $P(q)$ (blue line in Figure 6) without the usual $q^{-1}$ slope in the observed q-range. Using this form factor, determined from the fit of the last recorded patterns, it is possible to fit the entire experimental data $\left(I_{\text {micelle }}(q)\right.$ and $\left.I_{\text {Bragg }}(q)\right)$ from 150 seconds of reaction (before the Bragg peaks appearance) until the end of the measurement (Figure S2). This means that $I_{\text {micelle }}(q)$ can also be interpreted by cylindrical micelles, in agreement with a sphere to rod mechanism.

In conclusion, even if no great change of the scattering signal is detected in the $\mathrm{q}$ range between 0.016 and $0.06 \AA^{-1}$, the best fits (Figure S2) suggest a transition from non interacting ellipsoidal micelles at the beginning of the reaction to isolated cylindrical ones before the formation of the mesophase. Figure 7 summarizes the two possible mechanisms for the formation of SBA-3 materials using $\mathrm{CTA}^{+}$as structure directing agent. The "sphere to rod mechanism" which allowed fitting the entire set of data including the Bragg peaks is preferred to the "reservoir" one coming from a more qualitative point of view onto the scattering curves. 


\subsection{Formation mechanism of SBA-1: Structure factor in the cubic $\operatorname{Pm}^{\overline{3}}$ n systems}

In this section we will focus on the structural evolution of the SBA- $1 \mathrm{SiO}_{2}$ material with cubic $\mathrm{Pm}^{\overline{\mathbf{3}}} n$ (a 3D packing of isovolumetric polyhedral (spheroidal) micelles ${ }^{58}$ ) mesostructured phase obtained from $\mathrm{CTEA}^{+}$, for which the same corresponding liquid crystalline phase was never observed for the respective pure micellar systems in water. ${ }^{59,60}$ The specific case of CTPA ${ }^{+}$, which is also precursor of a $\mathrm{Pm}^{\overline{\mathbf{3}}} n$ mesophase, will be discussed separately in a dedicated note in Sect. 3.4 because of its atypical micellar shape behaviour.

First of all, using the same approach discussed for $\mathrm{CTA}^{+}$, the scattering curves of CTEA ${ }^{+}$ micelles in pure water (Figure 3) can be fitted using an ellipsoidal core-shell micelles model and best fits correspond to prolate ellipsoidal micelles with a weak ellipticity equals to 1.9 (Table S1). The core radius and the shell thickness are also equal to 16.3 and $2 \AA$, as it was observed for $\mathrm{CTA}^{+}$with a contrast term $\alpha=-0.3$. Even if a specific comparison with literature is not possible for $\mathrm{CTEA}^{+}$due to lack of reference data, one can reasonably expect a close similarity to $\mathrm{CTA}^{+}$micelles under dilute conditions, which is experimentally found here.

The time-resolved in situ study of the mesophases is shown in Figures 4 and 5. As shown in Figure 4, CTEA ${ }^{+}$systematically leads to the cubic $\mathrm{Pm}^{\overline{\mathbf{3}}} n$ phase, where the (200), (210) and (211) reflections in the XRD pattern are nicely resolved. The first diffraction peaks related to the formation of the cubic mesostructure are detected 100 seconds after the TEOS addition, while more than ten reflections of the cubic $\mathrm{Pm}^{\mathbf{3}} n$ phase are detected at the end of the reaction. Cell parameter of the cubic structure decreases, as expected, with reaction time from 100 to $98 \AA$. The characteristic and most interesting feature of the cubic system formation is the systematic presence of a strong broad interaction peak (FWHM $=0.03 \AA^{-1}$ ) which appears after about 200 seconds. 
Figure 5 presents chosen scattering curves taken at different reaction times. As for the $\mathrm{CTA}^{+}$ one, the contribution of the silica clusters with the $\mathrm{q}^{-2}$ slope at high $\mathrm{q}$ ( $>0.2 \AA$ ) is clearly increasing upon time. Scattering by the surface of TEOS droplets or of silica grains is also reported at low angle. For CTEA ${ }^{+}$based system, no significant change in the form factor of the micelles signal appears and thus no modification of the micelles shape or contrast induced by the addition of the silica precursor can be detected. In fact, the CTEA ${ }^{+}$micelles contribution to the scattered signal is strongly modified in terms of their structure factor contribution by the addition of the silica precursor. Indeed, the interaction peak with a maximum located at $0.15 \AA^{-1}$ appears on the SAXS patterns after 150 seconds of reaction. From these observations, we can deduce that the micelles are packed locally together without a well-defined long-range order. In such situation, it is not possible to analyze an eventual modification of the form factor contribution, as the structure factor $S(q)$ contribution is dominating the signal. However, considering the geometry of the end mesophase composed of two isovolumetric globular micelles, it seems reasonable to assume that for CTEA ${ }^{+}$, the interacting micelles essentially keep an ellipsoidal shape. The interaction peak is detected in the same q-range as the (200) (210) and (211) reflections of the $\mathrm{Pm}^{\mathbf{3}} n$ cubic phase which appears 100 seconds after the TEOS addition. Its intensity increases during the first 200 seconds in parallel with the growth of the diffraction reflections and then remains almost constant until the end of the measurement.

The qualitative observations made previously have been confirmed by fitting the scattering curves (Figure S3 and 8). The micelle contribution $I_{\text {micelle }}(q)$ characterized by a broad interaction peak can be modeled by a Percus-Yevick structure factor with an effective volume fraction. The curves have been fitted keeping constant the form factor and by adjusting the micelles structure factor to model the interaction peak. Thus, the effective Percus Yevick micelles volume fraction increases during the first 200 seconds of reaction until 0.5 and 
remains then almost constant (Figure S3.b). This high volume fraction shows that the flocs are very concentrated. For these two systems, TEOS addition to the micellar solution leads to an increase of the structure factor contribution and also to the appearance of a slope at low $\mathrm{q}$ value. Indeed, the appearance of a strong interaction peak and the increase of the intensity for $\mathrm{q}<0.016 \AA^{-1}$ may correspond to the formation of flocs of spherical micelles in strong interaction. The presence of an interaction peak is usually correlated to interactions between the scattering objects and is often encountered at high surfactant concentrations. Therefore we can explain this strong interaction contribution to the scattering signal by the formation of local concentrated aggregates of non ordered spherical micelles surrounded by silica species. The increase of the intensity observed at q values lower than $0.016 \AA^{-1}$ related to the scattering of bigger particles surface is also consistent with this phenomenon. The cubic ordered silica grains appear then from these flocs as described on Figure 8. As for the CTA ${ }^{+}$, the micelles could act as a reservoir of surfactant monomers, but in this case, no experimental data confirms this phenomenon since no transition of the micelles structure is necessary. Considering that Bragg reflections appear before the interaction peak at reaction times as low as $98 \mathrm{~s}$ (Figure 5), it is possible that that at this stage of the reaction, due to the low proportion of aggregated flocs, the contribution to the scattered signal of the structure factor is very low. The diffraction signal coming from the mesophase is more intense due to the better contrast induced by the silica species.

The broad interaction peak is only observed for the cubic system in this study and never for the hexagonal SBA-3 material. One could then argue that the formation of cubic systems needs an initial phase where micelles are in close contact via the silica oligomers. This could be confirmed from literature studies reported during the synthesis of SBA-16 materials (Im3m micellar cubic structure) prepared using block copolymer F108 as template and where a broad correlation peak has been reported. ${ }^{61}$ In conclusion, the proposed mechanism for the 
formation of SBA-1 materials is summarized on Figure 8 and is characterized by an intermediate phase of flocs of spherical micelles.

\subsection{Formation mechanism of CTA+-templated organically- modified mesostructured silica materials}

In Figure 2 we have shown the striking effect of the organic group on the hexagonal-to-cubic mesophase change with time in RTES/TEOS (where R stands for a general organic group, here methyl, phenyl or vinyl groups) systems in presence of $\mathrm{CTA}^{+}$. On the basis of the knowledge acquired on the previous study of pure hexagonal $\mathrm{CTA}^{+} / \mathrm{TEOS}$ and cubic $\mathrm{CTEA}^{+} / \mathrm{TEOS}$ systems, we will describe and discuss in more detail the crucial effect of the organic group in these complex systems.

Figure 9 presents selected scattering curves extracted from the three kinetics measurements of Figure 2 at different times of reaction. As previously reported for kinetics measurements done using TEOS as silica source, a sharp increase of the intensity from the very beginning of the kinetics is detected for the three systems due to the formation of small silica clusters. This contribution can be modeled again with a gyration radius of a 2-5 Ångstrom of these clusters. Before the appearance of the Bragg peaks of the 2D-hexagonal mesophase, the micelles contribution is very similar for the three systems, with a very broad hump (FWHM $=0.10 \AA^{-}$ ${ }^{1}$ ). Indeed, curves recorded at times 211s (MTES), 117s (VTES) and 113s (PTES) can be almost superimposed, as shown in Figure 9 and Figure S4. A careful analysis of these three curves (Figure S4) shows that this broad hump cannot be satisfactorily interpreted by a typical structure factor interaction peak, (like for SBA-1, in section 3.2.) whose average FWHM is generally not more than $0.03 \AA^{-1}$. For the organo-modified silicas, the hump can be very well fitted by a core/shell cylindrical form factor corresponding to non-interacting rod-like 
micelles. The parameters used for this form factor are very similar for the three systems (MTES, VTES, PTES in Figure S4). Very interestingly, this form factor is also close to the one employed to model the TEOS/CTA ${ }^{+}$system. The latest at $900 \mathrm{~s}$, where the hexagonal mesophase is already formed, can be modeled with the same form factor (blue line $P(q)$ in Figure 6) which shows the same broad hump with comparable FWHM. The fit parameters, $\mathrm{R}_{\mathrm{c}}=16.9 \AA, \mathrm{R}_{\mathrm{tot}}=23.0 \AA$ and $\alpha=-1.05 \pm 0.05$, are the same (Table in Figure 6 and Table in Figure S4). Moreover, the fit parameters for the TEOS/CTA ${ }^{+}$system at lower reaction time ( $t=150 \mathrm{~s}$ and free cylindrical micelles) are the same except for the contrast term $\alpha$, which is higher ( $\alpha=-0.8$ instead of $\alpha=-1.1$ ), as seen in Figure S2. In this case, the form factor does not present a hump anymore (Figure S2 and Figure S4, t= 150 s).

Recalling that the contrast term is $\alpha=\left(\rho_{\text {shell }}-\rho_{0}\right) /\left(\rho_{\text {core }}-\rho_{0}\right)$, where $\rho_{0}$, $\rho_{\text {shell }}$ and $\rho_{\text {core }}$ stand for the electronic density of, respectively, the solvent, the hydrophilic shell and the hydrophobic core, more negative $\alpha$-values correspond to larger amount of silica oligomers in the shell. For example, for organo-free TEOS/CTA ${ }^{+}$(Figure S2) system $\alpha$ decreases upon time and the $\alpha=$ 1.1 value is reached only after $600 \mathrm{~s}$ from TEOS addition. For organically-modified silicas, the same value is reached 6 times earlier ( $\mathrm{t}=150 \mathrm{~s}$ from precursor addition). As a consequence, adding an organosilane precursor has two effects: the amount of silica oligomers interacting with the micelles increases and condensation times are shorter. Moreover, these data also show that the sphere to rod transition mechanism occurs prior to the formation of the 2D-hexagonal mesophase, as previously observed for the $\mathrm{CTA}^{+}$system.

The second very important phenomenon concerns the hexagonal-to-cubic phase transition presented earlier (Figure 2) and which only occurs with $\mathrm{CTA}^{+}$in presence of the organicallymodified silica precursor. The cubic phase being composed of spherical micelles, a rod-tosphere transition must take place in the condensed phase at longer reaction times. To better understand these complex phenomena, it can be useful to recall two pieces of information: 
Goletto et al. ${ }^{14,15}$ have shown that, in the PTES/TEOS system, the cubic mesophase can only be obtained for specific molar ratios (1:4), thus demonstrating the importance of stoichiometry; meanwhile, advanced ${ }^{1} \mathrm{H}-{ }^{1} \mathrm{H}$ single-quantum double-quantum solid-state NMR experiments have proved the spatial proximity between the organic group and the surfactant polar head in the dried powder, $\left.{ }^{6,62}\right\}$ thus showing its presence at the micellar palisade. Even if NMR data tend to support the hypothesis that the organic group may induce a change in the effective g-parameter, thus provoking a change in the local micellar curvature, from low to high (rod-to-sphere), one cannot neglect condensation effects of silica at the micellar surface. In particular, an optimum amount of silica oligomers may be necessary to obtain the cubic mesophase and such parameter can be dependent on stoichiometry of the organicallymodified silanes. What the present results show is that the presence of the organicallymodified silanes tends to promote the oligomers condensation around the micelles and that it may be another mechanism for the increase of the curvature upon time within the material that induces the 2D hexagonal to cubic transformation.

\subsection{Unexpected micellar shape in $\mathrm{CTPA}^{+}$system}

In this last section, we highlight the unexpected micellar behavior of $\mathrm{CTPA}^{+}$micelles and their shape evolution with time upon TEOS addition. First of all, CTPA ${ }^{+}$is precursor of a cubic $\mathrm{Pm}^{\overline{\mathbf{3}}} n$ silica mesophase, as shown in Figure S.1, even if the peak resolution is rather poor if compared to CTEA ${ }^{+}$-based systems. In situ SAXS experiments (Figure 4 and Figure 5) confirm this assumption, as the Bragg diffraction peaks only start to be observed very late in the reaction time. As already observed before, a broad interaction peak is also systematically characteristic for the cubic phase and in the $\mathrm{CTPA}^{+}$system it starts to appear at about 170 seconds from TEOS addition. 
$\mathrm{CTPA}^{+}$has a similar structure to $\mathrm{CTA}^{+}$and $\mathrm{CTEA}^{+}$but a more volumetric polar head. According to simple considerations based on the $g$-parameter (Figure 1 and SI), one would expect an increase of about $40 \%$ in the polar head surface from $\mathrm{CTA}^{+}$to $\mathrm{CTPA}^{+}$and a consequent decrease of $\mathrm{g}$ from 0.32 for $\mathrm{CTA}^{+}$to 0.21 for $\mathrm{CTPA}^{+}$. Under these conditions, micelles composed of $\mathrm{CTPA}^{+}$are expected to be spherical but the absence of basic studies on the micellization behavior of this compound ${ }^{63}$ does not allow a clear-cut answer. For this reason, we have run SAXS experiments on the micellar solution of $\mathrm{CTPA}^{+}$under the same conditions as $\mathrm{CTA}^{+}$and $\mathrm{CTEA}^{+}$and we have followed the mesophase evolution upon addition of TEOS during the formation of SBA- 1 material. The unexpected results and related discussion are reported hereafter and in Figures 4 and 5.

First of all, the scattering curve of the $\mathrm{CTPA}^{+}$micelles in acidic water presents completely different features with respect to $\mathrm{CTA}^{+}$and $\mathrm{CTEA}^{+}$(Figure 3). A I(q) $\mathrm{q}^{-5 / 3}$ slope is detected at $\mathrm{q}$ values lower than $0.04 \AA^{-1}$ along with a $\mathrm{q}^{-1}$ slope in the q range of $0.04-0.08 \AA^{-1}$. This kind of signal is characteristic of the scattering by wormlike micelles without interactions. ${ }^{40 \text {, }}$ 42, 64 Such micelles can be described as a long flexible aggregate of surfactant molecules, similar to a polymer chain. It is characterized at different length scales by the following quantities: The contour length $L_{c o n t}$, the Kuhn length $b_{K u h n}$ (twice the persistence length) and the cross-section diameter. The dependence observed for q values less than $0.04 \AA^{-1}\left(\mathrm{I}(\mathrm{q}) \sim \mathrm{q}^{-}\right.$ ${ }^{5 / 3}$ for a chain in good solvent or $\mathrm{q}^{-2}$ for a Gaussian chain) corresponds to the scattering by the polymer chain and is related to the contour length. The contour length could be determined if a plateau at low q was reached but the q range used in this study does not allow it. For intermediate $\mathrm{q}$ values, the $\mathrm{q}^{-1}$ slope corresponds to a regime where the scattering is dominated by the fact that the micelles are locally rodlike. The corresponding Holtzer plot ${ }^{64,65}$ (Figure 3 inset) allows to estimate the Kuhn length $b_{\text {Kuhn }}=3.8 / q^{*}=100 \AA$ ) from the position $q^{*}$ of the cross-over between the $\mathrm{q}^{-5 / 3}$ and $\mathrm{q}^{-1}$ regimes. For $\mathrm{q}$ values larger than $0.1 \AA^{-1}$, the strong 
decrease in the intensity is related to the cross-section form factor contribution, as detailed in the following. Best fits can be obtained for wormlike micelles: a contour length fixed at $L_{\text {cont }}$ $=100000 \AA$ provides a Kuhn length of $100 \AA \AA$, an elliptic core-shell section of ellipticity 1.6 and a broadly smeared shell contrast as shown in Figures 3 and S5 and Table S.1. The overall diameter of the wormlike micelles is about $60 \AA$. In the cross-section profile (Figure S5), the electron density is equal to $280 \mathrm{e} / \mathrm{nm}^{3}$ in the middle of the core region and increases up to 370 $\mathrm{e} / \mathrm{nm}^{3}$ in the shell, as the solvent electron density value is known and equals $350 \mathrm{e} / \mathrm{nm}^{3}$. This profile confirms that the contrast is quite weak between the shell and the solvent for CTPA ${ }^{+}$. The parameters used to fit the micelles scattering curves are given in Figure S5 in supporting information.

With respect to CTPA ${ }^{+}, \mathrm{CTA}^{+}$and $\mathrm{CTEA}^{+}$, two systems having smaller polar heads $\left(65.5 \AA^{2}\right.$ for $\left(\mathrm{CH}_{3}\right)_{3} \mathrm{~N}^{+} \mathrm{Br}^{-}$and $80.0 \AA^{2}$ for $\left.\left(\mathrm{C}_{2} \mathrm{H}_{5}\right)_{3} \mathrm{~N}^{+} \mathrm{Br}^{-}\right)$(Figure 1), form globular micelles. The increase of the alkyl chain attached to the ammonium ion from $\mathrm{C}_{2} \mathrm{H}_{5}$ to $\mathrm{C}_{3} \mathrm{H}_{7}\left(100.0 \AA^{2}\right)$ seems to lead to an astonishing structural change to wormlike micelles. These observations are at first sight surprising since the increase of the size of the polar head should lead to an increase of the micelle curvature by the modification of the packing parameter, as commented before. Thus, since $\mathrm{CTA}^{+}$and $\mathrm{CTEA}^{+}$form ellipsoidal aggregates, micelles composed of $\mathrm{CTPA}^{+}$should also be ellipsoidal or spherical and not wormlike. Even if we are at the moment unable to provide a full explanation to this behavior, we can argue that the formation of wormlike micelles with this surfactant should be related to the consequent increase of the hydrophobicity of the polar head because of the propyl groups. This may lead to an increase of the effective hydrophobic volume of the surfactant and accordingly to an increase of the packing parameter $g$. But the main effect is more probably a reduction of the effective polar head, that may be attributed to an hydrophobic interaction between the propyl groups within the Stern layer of micelles, favored by the high ionic strength which decreases the 
electrostatic repulsion between adjacent polar heads and modifies the water structure around the aggregates.

The effect of the TEOS addition on the CTPA ${ }^{+}$micellar behavior is shown in Figures 4 and 5. The presence of the $\mathrm{q}^{-5 / 3}$ slope related to the wormlike structure of the micelles bothers the observation at low-q in the initial stages of the reaction. Nevertheless, the addition of the silica precursor induces at low $\mathrm{q}$ a transition from the $\mathrm{q}^{-5 / 3}$ dependence of the intensity as a function of q to a plateau, which is a sign of the decrease of the wormlike micelles signal contribution as a function of the reaction time (Figure S5). No modification of their form factor can be detected, and the $\mathrm{I}(\mathrm{q}) \sim \mathrm{q}^{-5 / 3}$ is observed in coexistence with a broad interaction peak from 150 to 500 s. This interaction peak, described in section 3.2, can be interpreted by flocs of concentrated micelles with no long range order. Unfortunately, it is impossible to derive what kind of micelles is present in these flocs (wormlike, spherical ...). It could be possible that a wormlike material is forming by direct templating of wormlike micelles but here we make the assumption that the interaction peak is rather related to the formation of flocs of spherical micelles considering the imminent formation of the cubic phase (see section 3.2.). After $800 \mathrm{~s}$, an additional Bragg peak is observed, meaning that the $P m^{\overline{\mathbf{3}}} n$ phase starts to form. Then the system follows the same behavior reported above for CTEA ${ }^{+}$, despite the fact that the Bragg peaks appear at longer time. In conclusion, the formation of wormlike micelles does not seem to influence the formation mechanism of the cubic mesophase. As depicted in Figure 8, we suggest that the wormlike micelles essentially act as a reservoir of surfactant molecules and do not act as a template for the final material. The true mechanism can actually be more complicated, as one could imagine specific interactions between wormlike micelles with silica oligomers. Further experiments are needed to confirm this behavior. 


\section{Conclusion}

The aim of this work was to understand the genesis of organo-modified mesoporous silica, based on time-resolved in situ SAXS experiments.

Our results show that predictions of the behavior based on simple assumptions provided by the $g$-parameter appears to be an oversimplified way to put in relationship the structure and self-assembly properties of a given surfactant. For these fast self-assembling systems, we find that transient states as well as coexisting phases within the synthesis medium are very often involved. Then the structure of the final hybrid material is the result of a complex balance between several events upon time and, as a result, a rich variety of different pathways towards the same final structure is observed. The presence of the organosilane influences essentially the second part of the kinetics and the pathway from the transient 2D-hexagonal phase towards the $\mathrm{Pm}^{\overline{\mathbf{3}}} n$ cubic phase. VTES and PTES give a similar behavior, with a clear structural transformation between the two mesophases. For MTES only, a broad interaction peak is observed prior to the appearance of the Bragg peaks of the $\mathrm{Pn}^{\overline{\mathbf{3}}} \mathrm{m}$ phase, in a similar way as for the CTEA ${ }^{+} / \mathrm{TEOS}$ and $\mathrm{CTPA}^{+} / \mathrm{TEOS}$ systems. In conclusion, we find that two pathways are possible for the formation of a cubic $\mathrm{Pm}^{\overline{3}} n$ mesophase, either after a structural transformation from a 2D-hexagonal mesophase $\left(\mathrm{CTA}^{+}\right.$, TEOS and addition of PTES or VTES) (Figure 10) or from concentrated flocs of spherical micelles $\left(\mathrm{CTEA}^{+}\right.$or $\mathrm{CTPA}^{+}$with TEOS) (Figure 8). For the CTA ${ }^{+} / \mathrm{TEOS} / \mathrm{MTES}$ system, it may be that the two pathways are coexisting.

The following behaviors have been found for organosilane-free materials.

1) For the TEOS/CTA ${ }^{+}$system, a 2D-hexagonal mesophase is obtained even if it is initially characterized by spherical micelles. For this reason one expects a sphere-to-rod transition identified by the presence of a typical $\mathrm{q}^{-1}$ slope in SAXS curves at low q. Here, such an 
evident sign of a $\mathrm{q}^{-1}$ slope is never found in the time-resolved experiments. Thanks to the accurate modeling described in this work, we are able to show that, as expected, a sphere-torod shape transition may be involved, as the micelle contribution just before precipitation can be modeled by a cylindrical form factor for which the specific micelle/solvent electron density contrast strongly contributes to the absence of the $\mathrm{q}^{-1}$ slope in the measured q-range (Figure 6 and S2). However, we cannot completely rule out a reservoir mechanism where spherical/ellipsoidal micelles would constitute the molecular source for the hexagonal phase (Figure 7).

2) In the $\mathrm{Pm}^{\overline{\mathbf{3}}} n$ systems obtained from $\mathrm{CTEA}^{+}$and $\mathrm{CTPA}^{+}$surfactants, a broad interaction peak $\left(\right.$ FWHM $<0.03 \AA^{-1}$ ) is observed before the appearance of the Bragg peaks of the $P m^{\overline{3}} n$ phase, and can be interpreted by an intermediate state made of flocs of spherical micelles (Figure 8), where the micelles are close to each other but not already packed on a cubic lattice. Interestingly, this intermediate state is never observed in the case of the CTA ${ }^{+}$-driven systems.

3) We observe, against the $g$-parameter logic, the formation of wormlike micelles in the pure $\mathrm{CTPA}^{+}$system instead of the expected spherical micelles. One possible interpretation is that the presence of three hydrophobic propyl groups reduces the effective polar head by the way of an hydrophobic interaction between them in the Stern layer, which can be promoted by the decrease of the electrostatic repulsion between adjacent surfactants and/or the change in the water structure due to the high ionic strength. Upon TEOS addition, the presence of a broad interaction peak can be interpreted by analogy with the CTEA ${ }^{+}$system by the formation of flocs of spherical micelles before the appearance of the Bragg peaks at long reaction times. This system provides a very good example in which the surfactant-silica oligomers interactions have a strong influence on the micellar and mesophase curvature. 
When organosilanes are mixed with TEOS and $\mathrm{CTA}^{+}$, an intermediate 2D-hexagonal phase is systematically observed before the formation of a $\mathrm{Pm}^{\overline{3}} n$ cubic phase. As already observed previously in other systems, ${ }^{43,44}$ a clear epitaxial relationship between the (10) 2D hexagonal planes and the (211) cubic planes is observed during this structural change in the case of PTES and VTES. Two important conclusions can be drawn:

1) The micelles contribution before the appearance of the 2D-hexagonal phase can be modeled by almost the same cylindrical form factor for the three systems (MTES, VTES and PTES) (Figure S4), confirming the sphere-to-rod mechanism to be possible. Moreover, this form factor is the same to the one employed to model the 2D-hexagonal phase for the TEOS/CTA ${ }^{+}$system (Figure 6), except for the value of the contrast term $\alpha$, which is more negative. This indicates that when the organosilane is added, the outer micellar shell is richer in silicate oligomers and comparison between reaction times show that this occurs mush faster (150 s vs. 600 s) for the organically-modified system.

2) Very interestingly, and most likely unexpectedly, the chemical nature of the organic group (Methyl-, Phenyl-, Vinyl-) has no specific influence on the initial stages of micellar shape evolution. To explain the hexagonal-to-cubic mesophase transition one could invoke the plausible influence of the organic group on the local micellar curvature, as, according to gparameter arguments, one expects a shift towards more spherical micelles, present in the cubic mesophase. Our study shows that the silicate amount and its condensation kinetics at the micellar surface is also strongly affected by the presence of the organosilane precursor and this parameter seems to play a major role on the local micellar curvature variations.

\section{Acknowledgments}

The authors would like to thank the Agence Nationale de la Recherche (ANR, program ANR08-BLAN-0189-01) and the C'Nano IDF program for the postdoc support of respectively 
Florentin Michaux and Sabine Manet, and SOLEIL Synchrotron facility (Saint-Aubin, France) for beam-time allocation. Mika Linden (University of Ulm, Germany), Sarah Tolbert and Andy Riley (University of California at Los Angeles, USA) are warmly acknowledged for helpful discussions.

\section{References}

[1] Stein, A.; Melde, B. J.; Schroden, R. C., Hybrid inorganic-organic mesoporous silicates - Nanoscopic reactors coming of age. Adv. Mater. 2000, 12, 1403-1419.

[2] Theme Issue: Hybrid Materials, Chem. Soc. Rev. 2011, 40.

[3] Sanchez, C.; Belleville, P.; Popall, M.; Nicole, L., Applications of advanced hybrid organic-inorganic nanomaterials: from laboratory to market Chem. Soc. Rev. 2011, 40, 696-753.

[4] Lim, M. H.; Blanford, C. F.; Stein, A., Synthesis and Characterization of a Reactive Vinyl-Functionalized MCM-41: Probing the Internal Pore Structure by a Bromination Reaction. J. Am. Chem. Soc. 1997, 119, 4090-4091.

[5] Kao, H. M.; Wu, J. D.; Cheng, C. C.; Chiang, A. S. T., Direct synthesis of vinylfunctionalized cubic mesoporous silica SBA-1. Micropor. Mesopor. Mater. 2006, 88, 319-328.

[6] Kao, H. M.; Liao, C. H.; Hung, T. T.; Pan, Y. C.; Chiang, A. S. T., Direct Synthesis and Solid-State NMR Characterization of Cubic Mesoporous Silica SBA-1 Functionalized with Phenyl Groups. Chem. Mater. 2008, 20, 2412-2422.

[7] Beck, J. S.; Vartuli, J. C.; Roth, W. J.; Leonowicz, M. E.; Kresge, C. T.; Schmitt, K. D.; Chu, C. T.-W.; Olson, D. H.; Sheppard, E. W.; McCullen, S. B.; Higgins, J. B.; 
Schlenker, J. L., A new family of mesoporous molecular sieves prepared with liqud crystal templates. J. Am. Chem. Soc. 1992, 114, 10834-10843.

[8] Huo, Q.; Margolese, D. I.; Ciesla, U.; Feng, P.; Gier, T. E.; Sieger, P.; Leon, R.; Petroff, P. M.; Schüth, F.; Stucky, G. D., Generalized synthesis of periodic surfactant/inorganic composite materials. Nature 1994, 368, 317-321.

[9] Huo, Q.; Margolese, D. I.; Stucky, G. D., Surfactant control of phases in the synthesis of mesoporous silica-based materials. Chem. Mater. 1996, 8, 1147-1160.

[10] Burkett, S. L.; Sims, S. D.; Mann, S., Synthesis of hybrid inorganic-organic mesoporous silica by co-condensation of siloxane and organosiloxane precursors. Chem. Commun. 1996, 1367-1368.

[11] Macquarrie, D. J., Direct preparation of organically modified MCM-type materials. Preparation and characterisation of aminopropyl-MCM and 2-cyanoethyl-MCM Chem. Commun. 1996, 1961-1962.

[12] Fowler, S. L.; Burkett, S. L.; Mann, S., Synthesis and characterization of ordered organo-silica-surfactant mesophases with functionalized MCM-41-type architecture Chem. Commun. 1997, 1769-1770.

[13] Han, L.; Terasaki, O.; Che, S., Carboxylic group functionalized ordered mesoporous silicas. J. Mater. Chem. 2011, 21, 11033-11039.

[14] Goletto, V.; Dagry, V.; Babonneau, F., One-pot synthesis of a cubic silicate phase functionalized with phenyl groups. Mater. Res. Soc. Symp. Proc. 1999, 576, 229-234.

[15] Goletto, V.; Imperor, M.; Babonneau, F., Phenyl-functionalized silicate mesophases with hexagonal or cubic symmetries: influence of synthesis parameters. In Nanoporous Materials II; Studies in Surface Science and Catalysis, Sayari, A.; Jaroniec, M.; Pinnavaia, T. J., Eds. Elsevier: Amsterdam, 2000; Vol. 129, pp 287-294. 
[16] Israelachvili, J. N.; Mitchell, D. J.; Ninham, B. W., Theory of Self-Assembly of Hydrocarbon Amphiphiles Into Micelles and Bilayers. J. Chem. Soc., Faraday Trans. 2 1976, 76, 1525-1568.

[17] Che, S.; Lim, S.; Kaneda, M.; Yoshitake, H.; Terasaki, O.; Tatsumi, T., The effect of counteranion on the formation of mesoporous materials under the acidic synthesis process. J. Am. Chem. Soc. 2002, 124, 13962-13963.

[18] Che, S.; Li, H.; Lim, S.; Sakamoto, Y.; Terasaki, O.; Tatsumi, T., Synthesis mechanism of cationic surfactant templating mesoporous silica under and acidic synthesis process. Chem. Mater. 2005, 17, 4103-4113.

[19] Ting, C. C.; Wu, H. Y.; Pan, Y. C.; Vetrivel, S.; Fey, G. T. K.; Kao, H. M., Sugarassisted synthesis of ordered and stable cubic mesoporous silica SBA-1. J. Phys. Chem. C 2010, 114, 19322-19330.

[20] Zana, R.; Frasch, J.; Soulard, M.; Lebeau, B.; Patarin, J., Fluorescence probing investigations of the mechanism of formation of organized mesoporous silica. Langmuir 1999, 15, 2603-2606.

[21] Patarin, J.; Lebeau, B.; Zana, R., Recent advances in the formation mechanisms of organized mesoporous materials. Curr. Opin. Colloid Interface Sci. 2002, 7, 107-115.

[22] Flodström, K.; Wennerström, H.; Alfredsson, V., Mechanism of Mesoporous Silica Formation. A Time-Resolved NMR and TEM Study of Silica-Block Copolymer Aggregation. Langmuir 2004, 20, 680-688.

[23] Ruthstein, S.; Frydman, V.; Goldfarb, D., Study of the Initial Formation Stages of the Mesoporous Material SBA-15 Using

Spin-Labeled Block Co-polymer Templates. J. Phys. Chem. B 2004, 108, 9016-9022. 
[24] Linton, P.; Rennie, A. R.; Alfredsson, V., Evolution of structure and composition during the synthesis of mesoporous silica SBA-15 studied by small angle neutron scattering. Solid State Sci. 2011, 13, 793-799.

[25] Teixeira, C. V.; Amenitsch, H.; Linton, P.; Linden, M.; Alfredsson, V., The role played by salts in the formation of SBA-15, an in situ Small angle $\mathrm{x}$ ray scattering/diffraction study. Langmuir 2011, 27, 7121-7131.

[26] Flodström, K.; Teixeira, V.; Amenitsch, H.; Alfredsson, V.; Linden, M., In situ synchrotron small-angle X-ray scattering/X-ray diffraction study of the formation of SBA-15 mesoporous silica. Langmuir 2004, 20, 4885-4891.

[27] Ruthstein, S.; Schmidt, J.; Kesselman, E.; Talmon, Y.; Goldfarb, D., Resolving intermediate solution structures during the formation of mesoporous SBA-15. J. Am. Chem. Soc. 2006, 128, 3366-3374.

[28] Grosso, D.; Balkenende, A. R.; Albouy, P. A.; Ayral, A.; Amenitsch, H.; Babonneau, F., Two-Dimensional Hexagonal Mesoporous Silica Thin Films Prepared from Block Copolymers: Detailed Characterization and Formation Mechanism. Chem. Mater. 2001, 13, 1848.

[29] Zholobenko, V. L.; Khodakov, A. Y.; Durand, D., Synchrotron X-ray diffractiondiffusion studies of the preparation of SBA-15 materials. Micropor. Mesopor. Mater. 2003, 66, 297-302.

[30] Khodakov, A. Y.; Zholobenko, V. L.; Impéror-Clerc, M.; Durand, D., Characterization of the initial stage of SBA-15 synthesis by in situ time resolved small-angle X-ray scattering. J. Phys. Chem. B 2005, 109, 22780-22790.

[31] Imperor-Clerc, M.; Grillo, I.; Khodakov, A. Y.; Zholobenko, V. L.; Durand, D., New insights into the initial steps of the formation of SBA-15 materials : an in situ small angle neutron scattering investigation. Chem. Commun. 2007, 834-836. 
[32] Linton, P.; Rennie, A. R.; Zackrisson, M.; Alfredsson, V., In Situ Observation of the Genesis of Mesoporous Silica SBA-15: Dynamics on Length Scales from $1 \mathrm{~nm}$ to 1 $\mu$ m. Langmuir 2009, 25, 4685-4691.

[33] Sundblom, A.; Oliveira, C. L. P.; Palmqvist, A. E. C.; Pedersen, J. S., Modeling in Situ Small-Angle X-ray Scattering Measurements Following the Formation of Mesostructured Silica. J. Phys. Chem. C 2009, 113, 7706-7713.

[34] Sundblom, A.; Oliveira, C. L. P.; Pedersen, J. S.; Palmqvist, A. E. C., On the Formation Mechanism of Pluronic-Templated Mesostructured Silica. J. Phys. Chem. C 2010, 114, 3483-3492.

[35] Manet, S.; Lecchi, A.; Imperor-Clerc, M.; Zholobenko, V. L.; Durand, D.; Oliveira, C. L. P.; Pedersen, J. S.; Grillo, I.; Meneau, F.; Rochas, C., Structure of the micelles of a non-ionic copolymer determined by SANS and SAXS. J. Phys. Chem. B 2011, 115, 11318-11329.

[36] Manet, S.; Schmitt, J.; Imperor-Clerc, M.; Zholobenko, V. L.; Durand, D.; Oliveira, C. L. P.; Pedersen, J. S.; Gervais, C.; Baccile, N.; Babonneau, F.; Grillo, I.; Meneau, F.; Rochas, C., Kinetics of the formation of 2D-hexagonal silica nano-structured materials by non-ionic block copolymer templating in solution. J. Phys. Chem. B 2011, 115, 11330-11344.

[37] Yang, B.; Jaber, R.; Edler, K. J., Silica-surfactant-polyelectrolyte film formation: evolution in the subphase. Langmuir 2012, DOI: 10.1021/la3014317.

[38] Baccile, N.; Teixeira, C. V.; Amenitsch, H.; Villain, F.; Linden, M.; Babonneau, F., Time-resolved in situ raman and SAXS experiments: from silica precursor hydrolysis to development of mesoscopic order in SBA-3 surfactant templated silica. Chem. Mater. 2008, 20, 1161-1172. 
[39] Zholobenko, V. L.; Khodakov, A. Y.; Impéror-Clerc, M.; Durand, D.; Grillo, I., Initial stages of SBA-15 synthesis: An overview. Advances in Colloid. Interf. Sci. 2008, 142, $67-74$.

[40] Menger, F. M.; Littau, C. A., Gemini surfactants: A new class of self-assembling molecules. J. Am. Chem. Soc. 1993, 115, 10083-10090.

[41] Baccile, N.; Laurent, G.; Bonhomme, C.; Innocenzi, P.; Babonneau, F., Solid-state NMR characterization of the surfactant-silica interface in templated silicas: acidic versus basic conditions. Chem. Mater. 2007, 19, 1343-1354.

[42] Pedersen, J. S.; Schurtenberger, P., Scattering Functions of Semiflexible Polymers with and without Excluded Volume Effects. Macromolecules 1996, 29, 7602-7612.

[43] Kamiya, S.; Tanaka, H.; Che, S.; Tatsumi, T.; Terasaki, O., Electron microscopic study of structural evolutions of silica mesoporous crystals: crystal-growth and crystaltransformation from p6mm to Pm3n with time. Solid State Sci. 2003, 5, 197-204.

[44] Che, S.; Kamiya, S.; Terasaki, O.; Tatsumi, T., The formation of cubic Pm3n mesostructure by an epitaxial phase transformation from hexagonal p6mm mesophase. J. Am. Chem. Soc. 2001, 123, 12089-12090.

[45] Agren, P.; Linden, M.; Rosenholm, J. B.; Schwarzenbacher, R.; Kriechbaum, M.; Amenitsch, H.; Laggner, P.; Blanchard, J.; Schüth, F., Kinetics of cosurfactantsurfactant-silicate phase behavior. 1. Short-chain alcohols. J. Phys. Chem. B 1999, 103, 5943-5948.

[46] Tiemann, M.; Goletto, V.; Blum, R.; Babonneau, F.; Amenitsch, H.; Linden, M., Insitu SAXS studies on the formation of silicates/surfactant mesophases with solubilized benzene under acidic conditions. Langmuir 2002, 18, 10053-10057.

[47] Tolbert, S. H.; Landry, C. C.; Stucky, G. D.; Chmelka, B. F.; Norby, P.; Hanson, J. C.; Monnier, A., Phase transformations in mesostructured silica/surfactant composites: 
surfactant packing and the role of charge density matching. Chem. Mater. 2001, 13, 2247-2256.

[48] Landry, C. C.; Tolbert, S. H.; Gallis, K. W.; Monnier, A.; Stucky, G. D.; Norby, P.; Hanson, J. C., Phase transformation in mesostructured silica/surfactant composite. Mechanisms for change and applications to materials synthesis. Chem. Mater. 2001, 13, 1600-1608.

[49] Reiss-Husson, F.; Luzzati, V., The structure of the micellar solutions of some amphiphilc compounds in pure water as determined by absolute small angle X-ray scattering techniques. J. Phys. Chem. 1964, 68, 3504-3511.

[50] Hayter, J. B.; Penfold, J., Determination of micelle structure and charge by neutron small-angle scattering. Colloid \& Polymer Sci. 1983, 261, 1022-1030.

[51] Berr, S. S.; Caponetti, E.; Johnson, J. S.; Jones, R. R. M.; Magid, L. J., Small angle neutron scattering for hexadecyltrimethylammonium bromide micelles in aqueous solutions. J. Phys. Chem. 1986, 90, 5766-5770.

[52] Quirion, F.; Magid, L. J., Growth and counterion binding of cetyltrimethylammonium bromide aggregates at $25^{\circ} \mathrm{C}$ : A neutron and light scattering study. J. Phys. Chem. 1986, 90, 5435-5441.

[53] Berr, S. S., Solvent isotope effect on alkyltrimethylammonium bromide micelles as a function of alkyl chain length. J. Phys. Chem. 1987, 91, 4760-4765.

[54] Quirion, F.; Drifford, M., From cetyltrimethylammonium bromide micelles to 2Butooxyethanol aggregates stabilized by cetyltrimethylammonium bromide molecules: A small angle neutron scattering study. Langmuir 1990, 6, 786-791.

[55] Goyal, P. S.; Dasannacharya, B. A.; Kelkar, V. K.; Manohar, C.; Srinivasa Rao, K.; Valaulikar, B. S., Shapes and sizes of micelles in CTAB solutions. Physica B 1991, 174, 196-199. 
[56] Aswal, V. K.; Kohlbrecher, J.; Goyal, P. S.; Amenitsch, H.; Bernstoff, S., Counterion condensation on charged micelles in an aqueous electrolyte solution as studied with combined small angle neutron scattering and small angle x-ray scattering. J. Phys. Condens. Matter 2006, 18, 11399-11410.

[57] Hargreaves, R.; Bowron, D. T.; Edler, K., Atomistic Structure of a Micelle in Solution Determined by Wide Q-Range Neutron Diffraction. J. Am. Chem. Soc. 2011, 133, 16524-16536.

[58] Sakamoto, Y.; Kaneda, M.; Terasaki, O.; Zhao, D. Y.; Kim, J. M.; Stucky, G. D.; Shin, H. J.; Ryoo, R., Direct imaging of the pores and cages of three-dimensional mesoporous materials. Nature 2000, 408, 449-453.

[59] Rendall, K.; Tiddy, G. J. T.; Trevethan, M. A., Optical microscopy and nuclear magnetic resonance studies of mesophases formed at compositions between hexagonal and lamellar phases in sodium n-alkanoate + water mixtures and related surfactant systems. J. Chem. Soc., Faraday Trans. 1 1983, 79, (637-649).

[60] Blackmore, E. S.; Tiddy, G. J. T., Phase behaviour and lyotropic liquid crystals in cationic surfactant-water systems. J. Chem. Soc., Faraday Trans. 2 1988, 84, 11151127.

[61] Flodström, K.; Wennerström, H.; Teixeira, C. V.; Amenitsch, H.; Linden, M.; Alfredsson, V., Time-resolved in situ studies of the formation of cubic mesoporous silica formed with triblock copolymers. Langmuir 2004, 20, 10311-10316.

[62] Bonhomme, C.; Coelho, C.; Baccile, N.; Gervais, C.; Azaïs, T.; Babonneau, F., Advanced Solid State NMR Techniques for the Characterization of Sol-Gel Derived Materials. Acc. Chem. Res. 2007, 40, 738-746 
[63] Di Michele, A.; Brinchi, L.; Di Profio, P.; Germani, R.; Savelli, G.; Onori, G., Effect of head group size, temperature and counterion specificity on cationic micelles. $J$. Colloid Interface Sci. 2011, 358, 160-166.

[64] Dreiss, C. A., Wormlike micelles: where do we stand? Recent developments, linear rheology and scattering techniques. Soft. Matter. 2007, 3, 956-970.

[65] Holtzer, A., Interpretation of the angular distribution of the light scattered by a polydisperse system of rods. J. Polym. Sci. 1955, 17, 432-434. 